\title{
25 Research Square \\ Paediatric Enteric Fever in Brussels: a case series over 16 years
}

Valbona Selimaj Kontoni ( $\nabla$ valbona.selimaj23@gmail.com )

Saint-Jean Clinic: Clinique Saint-Jean https://orcid.org/0000-0002-6224-2705

\section{Philippe Lepage}

HUDERF: Hopital Universitaire des Enfants Reine Fabiola

\section{Marc Hainaut}

CHU Saint-Pierre: Centre Hospitalier Universitaire Saint-Pierre

Véronique Yvette Miendje Deyi

ULB: Universite Libre de Bruxelles

Wesley Mattheus

Sciensano

David Pace

Mater Dei Hospital

\section{Research Article}

Keywords: typhoid fever, children, Salmonella

Posted Date: July 29th, 2021

DOI: https://doi.org/10.21203/rs.3.rs-553247/v1

License: (c) (i) This work is licensed under a Creative Commons Attribution 4.0 International License. Read Full License 


\section{Abstract}

\section{Background}

Enteric fever (EF) is a major public health problem and a witness of the global health disparities. It is caused by Salmonella enterica serovar typhi (Salmonella ser. Typhi) and Salmonella enterica serovar Paratyphi A,B,C (Salmonella ser. Paratyphi) and is estimated to infect 12-26 million persons yearly.

Paediatric data on enteric fever in Europe are scarce. A case series of EF was analysed to describe the clinical presentation, laboratory characteristics and diagnostic challenges identified in a paediatric population in Brussels.

\section{Methods}

We performed a retrospective study of all lab-confirmed cases of enteric fever in children aged 0-15 years at two Brussels teaching hospitals, between January 2005 and December 2020.

We reviewed age, gender, travel history, consultations before diagnosis, hospitalisation duration, clinical symptoms and laboratory findings.

\section{$\underline{\text { Results }}$}

There were 34 positive isolates of S.typhi and S.paratyphi: 31 patients had positive blood culture, 1 patient had positive bone drainage, 2 patients had positive stool culture (one was excluded for missing data). There were 20 girls(60\%). Median age was 3.5 years (range 5 months to 14 years). Travel to EF endemic areas was present in $55 \%$ of patients. Diagnosis was delayed in $80 \%$ of children. Eosinopenia was present in $93 \%$ of the cohort. The patients had not received any preventive travel education or vaccination.

\section{Conclusions}

Enteric fever poses diagnostic challenges to clinicians. Eosinopenia in a febrile patient coming from the tropics should raise suspicion of EF. Travelers to endemic areas, should be better educated about EF risks and typhoid fever vaccination must be promoted.

\section{What Is Known}

- Enteric fever is a vaccine preventable disease and a global public health problem.

- Data on enteric fever for children living in developed countries is scarce.

\section{What is new}

- We report that the diagnosis of enteric fever is very challenging in non-endemic settings.

- The majority of children affected may have diagnostic delay due to un-specific clinical findings. 
- Eosinopenia is a reliable biologic finding at the enteric fever affected children.

- Travelers to endemic areas must be educated on EF risks.

\section{Introduction}

Typhoid and paratyphoid fever, commonly referred to as "enteric fever" are systemic infections caused by the Gram-negative bacilli Salmonella enterica serovar Typhi (Salmonella ser. Typhi) and Salmonella enterica serovar Paratyphi A, B, C (Salmonella ser. Paratyphi). Enteric fever is found only in humans and the transmission occurs by faecal-oral route [1] either by an acutely infected person, by a chronic carrier shedding the bacteria, or by the consumption of contaminated food or water[2]. Enteric fever is endemic in countries with limited access to safe water and sanitation systems. Considered by the World Health Organization (WHO) to be a major global public health problem, a significant burden of the disease is carried by mid- to low-income communities[3], the greatest weight being shared by South Asia and subSaharan Africa[4], which is a characteristic example of global inequality in health, prevention and care.

In the last few decades, the annual disease burden of enteric fever has been estimated to be between 11.9 and 26.9 million cases[4]. This disease claims 128,000 to 216,000 lives annually[5] and the majority of deaths are found in children aged 5-9 years. Data gathered from populations in more developed countries show fewer instances of typhoid outbreaks, with an approximate incidence rate ranging between $0.42-0.68 / 100.000$ persons which is disproportionately much less than the estimated 200$700 / 100,000$ individuals affected during typhoid outbreaks in South Asia and sub-Saharan Africa[6]. The rate of disease in the resource rich countries has fallen drastically particularly after the Second World War with the installation of sanitation plants and safe-water pipelines and autochthonous enteric fever occurs only sporadically[7]. This is the reason why, in Europe, as in other industrialised countries, enteric fever is mostly imported by travellers visiting the endemic areas, either as tourists or visiting relatives and friends (VRF)[8], [9].

Enteric fever is a fascinating, complex and life threatening disease[10] that behaves as a chameleon in terms of its capacity to mimic other infections in the way it presents [11]. Gaps in its diagnosis and its early recognition remain a pragmatic challenge for physicians all around the world, particularly in the developed countries where EF is very uncommon. Published data on enteric fever in low and middleincome countries exist, although often considered as underestimated, but paediatric data on EF in Europe are scarce. In this context, a case series of enteric fever was analysed to describe the clinical, biological and microbiological characteristics as well as the preventative, diagnostic and treatment challenges identified in a paediatric population living in Brussels.

\section{Methods}

Study Design 
We carried out a retrospective observational study of all cases of typhoid and paratyphoid fever in children aged 0-15 years. The study was undertaken across two University teaching hospitals in Brussels, namely at the Centre Hospitalier Universitaire Saint Pierre and Hôpital Universitaire des Enfants Reine Fabiola, between January 2005 and December 2020.

Permission to perform the study was granted by the respective University Hospitals' Ethical Boards. Within this remit, we sourced all lab-confirmed cases of Salmonella ser. Typhi and Salmonella ser. Paratyphi within January 2005 to December 2020, from the two Teaching Hospitals in Brussels, Belgium.

\section{Case Definition}

Cases were included if Salmonella ser. Typhi or Salmonella ser. Paratyphi was isolated from the blood culture, other sterile sites (such as cerebro-spinal fluid, bone marrow and bone aspirates), or from the stool culture in a context of a febrile syndrome. All the isolates were submitted to the Belgian National Centre of Reference for Salmonella for serotyping. All the samples were tested for antibiotic susceptibility following standard microbiologic procedures.

Information on the evolution of the fever and data regarding the immediate follow-up with patients were obtained through electronic medical records.

We looked at variables such as age, gender, history of travel, time elapsed after traveling from/ to enteric fever endemic areas; diagnostic challenges, such as number of consultations prior to diagnosis, length of hospitalisation; clinical features such as fever, cough, headache, gastrointestinal symptoms; and biological findings such as Haemoglobin, C -Reactive Protein (CRP), White Blood Cell (WBC) counts, Neutrophils, Lymphocytes, Eosinophils and Platelet counts, sodium concentration and concentration of Liver enzymes such as Alanine Aminotranspherase, (ALT) and Aspartate Aminotranspherase (AST).

We defined absolute eosinopenia when the eosinophil count was $0 / \mu \mathrm{L}$, eosinopenia when the eosinophils were less than $50 / \mu \mathrm{L}$; leukopenia when values of WBC were less than $5000 / \mu \mathrm{L}$ and thrombocytopenia when the values of platelets were less than $150000 / \mu \mathrm{L}$.

\section{Results}

Salmonella ser. Typhi and Salmonella ser. Paratyphi was isolated in 34 patients between January 2005 and December 2020. One patient with a positive stool culture, had missing data and was excluded from the study. The records of the other 33 patients were retrieved. There were 31 patients identified with positive blood cultures; 1 had only positive stool cultures and for one patient S.paratyphi grew from pus drained from a bone(in a context of osteomyelitis). Within the case series, 18/33(55\%) had positive stool cultures.

Among the isolates, 25 were Salmonella ser. Typhi and 8 Salmonella ser. Paratyphi: one Salmonella ser. Paratyphi A, three Salmonella ser. Paratyphi B, two Salmonella ser. Paratyphi C and one strain was S.paratyphi untypable. (Appendix 1.) 
More than half of the patients (20/33 60\%) were females (Table 1$)$. The median age at diagnosis was 3. 5 years (range 5 months to 14 years). More than half of our patients $(18 / 33,55 \%)$ had a history of recent travel to Africa and South Asia. Among the patients who travelled from Brussels, eleven were visiting relatives and friends (VRF). Two patients migrated to Belgium, and two children were African residents visiting relatives in Belgium.

Table 1

Demographic Characteristics

\begin{tabular}{|lc|}
\hline Age median, 25th and 75th percentile (years) & $\mathbf{3 . 5 ( 2 . 3 - 8 )}$ \\
\hline Gender & $20(60 \%)$ \\
\hline Females & $13(40 \%)$ \\
\hline History of travel & $18(55 \%)$ \\
\hline Travel to South Asia and Africa (number- percentage) & $7(21 \%)$ \\
\hline Contact with travellers from South Asia and Africa (number, percentage) & $7(21 \%)$ \\
\hline Unknown acquisition (number, percentage) & $16(48 \%)$ \\
\hline Ethnic origin & $14(42 \%)$ \\
\hline African origin patients (number, percentage)* & $2(6 \%)$ \\
\hline Asian origin patients (number, percentage)* & $1(3 \%)$ \\
\hline European origin patients (number, percentage)* & \\
\hline Middle East & \\
\hline $\begin{array}{l}\text { The countries of origin were Pakistan, India, Bangladesh, Morocco, Angola, Uganda, Guinea, Congo, } \\
\text { Saudi Arabia, Poland and Belgium. }\end{array}$ \\
\hline
\end{tabular}

Three children (9\%) had been in contact with a traveller affected by gastro-intestinal symptoms. Two other patients were in contact with relatives who had travelled recently to Africa, but were asymptomatic. Two patients participated in an international wedding and had a simultaneous onset of typhoid (together with two other members of the extended family (all present for the celebrations). For seven patients of our cohort, travel or history of close contact with a traveller to enteric fever endemic areas was not recorded. The time of potential exposure ranged from 0 to 60 days (from the date of travel to the return to Belgium).

The majority of our patients $26(78 \%)$ had visited the emergency room or attended a physician more than once: on average 2.5 times (with a range of 2-4 previous visits per patient) before admission to hospital, while only seven patients $(21 \%)$ were immediately admitted. We found that among the 33 patients affected by enteric fever, 31 were admitted and given intravenous antibiotics, while 2 patients were 
treated empirically with oral antibiotics with a close follow up by the medical staff. The mean hospital stay duration was eight days (with a range of 2 to 23 days).

We found that only one patient in our cohort had received typhoid fever vaccination before travelling to Africa (18 months prior to his typhoid fever episode). All other children had no recorded history of typhoid vaccination or consultation at the travel clinic.

The large majority of our patients; $29 / 33$ (87\%) had no underlying medical problems before being diagnosed with enteric fever. One was HIV positive and had stopped antiretroviral treatment prior to the typhoid fever episode and another child had sickle cell disease. Both of these children were newly arrived migrants to Belgium. One patient had Glucose 6 Phosphate Dehydrogenase (G6PD) Deficiency fortuitously discovered in the febrile course of his typhoid fever episode and one patient had a ventriculoperitoneal shunt.

The most common clinical symptoms were: fever, diarrhoea and vomiting, abdominal pain, unproductive cough and headache (Table 2). Rose spots and hepatosplenomegaly were each described in almost $10 \%$ of the cases.

Table 2

Presenting Clinical Symptoms

\begin{tabular}{|ll|}
\hline Clinical Symptoms & Number, Percentage \\
\hline Fever & $33,(100)$ \\
\hline Diarrhoea & $23,(69)$ \\
\hline Constipation & $5,(15)$ \\
\hline Abdominal pain & $25,(75)$ \\
\hline Rose spots & $3,(9)$ \\
Headache & $7,(21)$ \\
\hline Cough & $12,(36)$ \\
\hline Hepatosplenomegaly & $3,(9)$ \\
\hline
\end{tabular}

One patient developed Hemophagocytic Lymphohistiocytosis (Appendix 2.) and another patient experienced haemolytic anaemia over G6PD deficiency during the course of their enteric fever. Both of them were admitted to the intensive care unit. One patient had a weight loss of $14 \%$; one patient presented with lateralized complex febrile seizures and one developed multifocal osteomyelitis. One case relapsed after one month. This patient had received a shortened antibiotic course. In this case, the susceptibility profile of the isolate was identical to that of the first episode and the patient was successfully treated. Two children developed ileitis, one developed cholecystitis and intussusception, and another one had hepatitis and biliary cholelithiasis. 
In addition, we had a cluster in one family who hosted a relative resident in Angola. The first child affected (aged one) developed typhoid two weeks after the contact. The eldest (aged seven) and a close cousin (aged four) became febrile and had positive blood cultures one month after contact. All of them recovered fully. In a similar case, we recorded a second cluster of four patients, two adults and two children (ages 11 and 12). They all developed typhoid between 7-15 days after participating in a wedding where many people coming from Morocco had attended.

Blood investigations (Table 3) showed that 23 (69\%) patients had absolute eosinopenia with an eosinophil count of 0 per microscopic field. In total 31 (93\%) patients had less than 50 eosinophils; one patient had 60 eosinophils and one patient had 350 eosinophils. Leukopenia was present in 3 (9\%) patients on admission; 4(12\%) presented with leucocytosis, the rest $-26(79 \%)$ had a normal white blood cell count (overall range 3500-29420/ $/ \mathrm{l}$.). On admission, 3(9\%) patients had thrombocytopenia, 20 (60\%) patients were hyponatraemic, and 24 (73\%) had elevated levels liver function tests.

The antibiograms performed, showed that $6(18 \%)$ of strains were resistant to Ampicillin; 7 strains of Salmonella ser. Typhi and 2 strains of Salmonella ser. Paratyphi were resistant to Ciprofloxacin; 4 strains of Salmonella ser. Typhi (13\%) were nonsusceptible to trimethoprim-sulfamethoxazole. All the isolates were susceptible to third generation cephalosporins. 
Table 3

Haematologic and Biochemical Findings of Children Affected with Enteric Fever (median, 25th and 75th percentile)

\begin{tabular}{|ll|}
\hline Haemoglobin $\mathrm{g} / \mathrm{dL}$ & $11.6(10,6-12,2)$ \\
\hline White blood cell count $\left(10^{3} / \mu \mathrm{L}\right)$ & $8.4(6,6-11,4)$ \\
\hline Neutrophils absolute value $\left(10^{3} / \mu \mathrm{L}\right)$ & $4.65(3,58-6,17)$ \\
\hline Neutrophils percentage $(\%)$ & $51.6(41-60)$ \\
\hline Lymphocytes absolute value $\left(10^{3} / \mu \mathrm{L}\right)$ & $4.02(1,7-4,9)$ \\
\hline Lymphocytes percentage $(\%)$ & $38.7(25,4-48,7)$ \\
\hline Eosinophils count $\left(10^{3} / \mu \mathrm{L}\right)$ & $0(0-350)$ \\
\hline Eosinophils percentage $(\%)$ & $0(0-0.015)$ \\
\hline Platelet count $\left(10^{3} / \mu \mathrm{L}\right)$ & $273(170-365)$ \\
\hline CRP $(\mathrm{mg} / \mathrm{L})$ & $44(25-92)$ \\
\hline AST $(\mathrm{UI} / \mathrm{L})$ & $89(69-185)$ \\
\hline ALT $(\mathrm{UI} / \mathrm{L})$ & $65.5(41-132)$ \\
\hline BUN $(\mathrm{mg} / \mathrm{dL})$ & $24(14-28)$ \\
\hline Creatinine $(\mathrm{mg} / \mathrm{dL})$ & $0.4(0,3-0,48)$ \\
\hline Na+ (mEq/L) & $133(124-140)$ \\
\hline
\end{tabular}

\section{Discussion}

This study took place in Brussels, a capital city with a population of $1,300,000$ people, which it is home to residents from over 182 different national backgrounds [12], (Brussels Institute for Analysis and Statistics, 2018), and thirty percent of its habitants were born abroad (13). The University centres where our study was carried out, are two significant public institutions, known to treat particularly a large number of immigrant population compared to other local hospitals.

Although enteric fever is a rare occurrence in Belgium, in the era of globalisation and with the accessibility to long distance travel coupled with multicultural societies, the medical community should be more aware of the diagnostic challenges surrounding enteric fever, especially in a traveller returning from countries hyperendemic for enteric fever. It is important to note, that one fourth of our patients acquired enteric fever locally without a history of travel abroad. In addition, our study found that the majority of our patients were originally coming from areas highly endemic to enteric fever. This correlates also with other clinical studies[13], [14] as well as with a recent paediatric study from France[15]. In the 
city of Brussels, there are over seven travel clinics recognized as international vaccination centres by the WHO. They serve all the citizens intending to travel around the world. However, out of the whole cohort, only one patient had been immunized 18 months before he contracted the disease. None of the patients that had travelled recently was vaccinated, or had received prior education on enteric fever prevention measures. It is noteworthy that Belgium has a social health care system where all classes of the society have equal access and benefit. Based on the findings of our study, we believe that better education and prevention measures should be given to the future travellers particularly to those visiting relatives and friends[16].

Furthermore, the last WHO's position[17] paper on typhoid, encourages heavily-burdened countries to add a new generation Typhoid Conjugated Vaccine (TCV) to their national vaccination program as more efficient in terms of immunogenicity and duration of protection. Based on these recommendations, younger children starting from the age of six months can benefit from typhoid immunization. Therefore, immunization, coupled with clear education measures on hygiene, safe water and food consumption can be effective on limiting the incidence of enteric fever.

We found that more than the half of our enteric fever affected patients were below 5 years of age (appendix 1), and this correlates with the findings of Sinha et al. [18] although the most recent global estimates [5] showed a peak in the incidence of enteric fever and the highest mortality rates in children aged 5 to 9 years. However, the authors note that in the heavy burdened countries children are the mostly affected compared to a broader age distribution in low incidence regions. Our study overviewed a 16-year period in a city with low incidence rates of enteric fever. Therefore, it is difficult to generalize these agerelated findings in different socio-economic contexts.

All our patients fully recovered from their enteric fever episode and there were no fatalities similar to the outcome in other industrialised setting [4], [15], [19], but despite all these favourable elements, and the small sample cohort, we were able to identify serious complications. We found that the majority of our patients came to the emergency room or were examined by a physician in average two-three times before diagnosis or admission. They all were initially diagnosed with viral infection and despite sometimes repeated complementary blood exams, the findings were inconclusive and patients were sent home without an appropriate diagnosis. Similar findings on misdiagnosis were also described earlier [20], [21], and this confirms the diagnostic difficulties the clinician faces confronting enteric fever as one of the most challenging febrile systemic diseases, provided the few alarming clinical symptoms, the very low inflammatory markers and the low sensitivity of blood cultures[22]. A recent review by Mogasale et al. (18) showed that only $61 \%$ of true positive Salmonella ser. Typhi cases were detected through blood cultures meaning that even through blood cultures, two out of five enteric fever affected patients were not detected. Although scientific efforts have been done through the years to achieve better diagnostic tools of enteric fever with typhoid rapid tests, data from the last Cochrane review study[23] suggest that the diagnostic tools on better tests have not managed to replace blood cultures and cultures of bone marrow aspirates in the establishment of a diagnosis of enteric fever. 
On analysing this cohort, we found that one of the most consistent hematologic features in our patients was the presence of eosinopenia. Thirty-one of our patients were eosinopenic with twenty-one of them having no eosinophils counted during their bacteriaemic phase.

Numerous studies have reached similar conclusions on the predominant presence of this hematologic parameter in patients affected by enteric fever [20], [24], [25] as well as in cases of sepsis[26] [27], as a positive discriminator of the enteric fever diagnosis. In this case series, among all the other hematologic and biochemical parameters analysed, eosinopenia, together with consistent clinical features, is suggestive of enteric fever. That is why, although enteric fever is not the only disease that causes eosinopenia [28], we believe that its presence must raise suspicion of enteric fever in case of prolonged fever. Importantly, we found low inflammatory markers (median CRP: $44 \mathrm{mg} / \mathrm{L}$ ) for bacteriaemic children which it is an important finding for all the clinicians evaluating febrile children returning from the tropics[29].

We did not have any Multi Drug-Resistant (MDR) strains in this 33-case cohort. However, $20 \%$ of the strains were resistant to Ampicillin and Ciprofloxacin. In Belgium, results from the Centre National de Reference de Salmonella et Shigella (CNRSS) show a low resistance to ciprofloxacin of less than $3 \%$. Furthermore, recent data[30], [31] testify to the emergence of Multi-Drug Resistant (MDR) strain which are found to be resistant to ampicillin, chloramphenicol and trimethoprim-sulfamethoxazole as well as the extremely drug-resistant strands (XDR); the Haplo 58 clone Salmonella ser. Typhi [31] known for expressing resistance to the five first line treatments of enteric fever ampicillin, chloramphenicol, trimethoprim-sulfamethoxazole, fluoroquinolones and third-generation cephalosporins. These data are a paradigm of the increasing difficulties in enteric fever treatment and a plea for the implementation of preventive measures.

We could identify from our cohort that all our patients had high grade fever of over $38.1^{\circ} \mathrm{C}$; the majority of patients had gastro-intestinal symptoms as is the presentation in other parts of the world[16] albeit sometimes confounded with upper respiratory symptoms such as unproductive cough. We observed that the older patients presented with headaches. This complaint is one of the most commonly found in adults as described by the human challenge study [32]; possibly this symptom could also occur in infants, but would be difficult to identify given the lack of verbal communication at this age.

All our patients fully recovered from their enteric fever episode and there were no fatalities similarly with the outcome in other industrialized settings[33].

Our study may not have the necessary sample size to allow generalisation especially in low living standard countries, that face major health care challenges; i.e., eighty seven percent of our patients were living in a European setting, without confounders of malnutrition, with easy access to health care facilities, and almost all of them were admitted in average for 8 days and had numerous complications. Importantly, eighty percent of our patients had a delay in diagnosis and went unrecognized. We believe that our observations in this case series have a particular added value by demonstrating the clinical 
features in children with enteric fever, proving also that challenges remain high regardless if we are in Karachi, Kigali or Brussels.

Clinicians should be aware of the diagnosis of enteric fever and its huge potential of causing disease. We report that unspecific clinical and biological findings were misleading and enteric fever diagnosis was delayed in more than three quarters of our cohort patients, which constitutes an importantly high proportion of cases. Blood cultures should be drawn repeatedly at the ER and at the ward independently of their low sensitivity in patients with prolonged fever. Enteric fever should be high on the list of differential diagnoses, especially in the presence of eosinopenia both in the returning traveller as well as in the patient with a prolonged fever particularly in a multicultural society. Lastly, we found that $93 \%$ $(31 / 33)$ of our patients originated from enteric fever endemic areas. It is therefore important to identify the origin of a patient as a risk factor for enteric fever occurrence and properly educate these patients before travelling to their home country.

Our case series has some limitations since, besides having a small sample size, some of the medical records on exposure and recent travel data of our patients were incomplete and we were fully dependent on physician's records. Lastly, the laboratory data were not exhaustive and homogenous for all the patients.

\section{Conclusions}

Enteric fever poses a diagnostic challenge to clinicians working in non-endemic areas.

However, it is pertinent to consider enteric fever when examining febrile returning travellers, as well as patients with prolonged fever, particularly in a multicultural society. Blood cultures should be drawn repeatedly to increase sensitivity. The presence of eosinopenia in a febrile patient coming from the tropics or subtropics, or in the differentials of prolonged fever should raise suspicion of enteric fever. Typhoid and paratyphoid fever in children may occur with normal white blood cell count or hyperleukocytosis. Efficient diagnostic methods for enteric fever are necessary to better tackle this challenging disease. Travelers to endemic areas, particularly the visiting relatives and friends should be better educated about enteric fever risks. Furthermore, typhoid fever vaccination for travellers, including children, intending to visit endemic areas should be promoted.

\section{Abbreviations}

$\mathrm{EF}$

Salmonella ser. Typhi

Salmonella ser. Paratyphi

VRF
Enteric fever

Salmonella enterica serovar typhi

Salmonella enterica serovar paratyphi

Visiting Relatives and Friends 


\section{Declarations}

\section{Funding: N/A}

Conflicts of Interest : No conflicts of interests to disclose

\section{Availability of data and material: available}

\section{Code availability: N/A}

\section{Authors contributions:}

Material preparation, and analysis were performed by Valbona Selimaj Kontoni. Philippe Lepage, Marc Hainaut and Veronique Yvette Miendje Deyi made possible the data collection. The first draft of the manuscript was written by Valbona Selimaj Kontoni. David Pace critically revised the manuscript. Philippe Lepage, Marc Hainaut and Veronique Yvette Miendje Deyi and Wesley Mattheus commented on previous versions of the manuscript. All authors read and approved the final manuscript.

Acknowledgments and special thanks. We would like to thank very much Dr Tessa Goetghebuer for her valuable comments and advice.

\section{References}

1. Linam WM, Gerber MA, “Changing epidemiology and prevention of Salmonella infections," Pediatr. Infect. Dis. J., 2007

2. Mogasale VV, Ramani E, Mogasale V, Park JY, Wierzba TF, "Estimating Typhoid Fever Risk Associated with Lack of Access to Safe Water: A Systematic Literature Review," J. Environ. Public Health, 2018

3. Kim J-H, Mogasale V, Im J, Ramani E, Marks F, "Updated estimates of typhoid fever burden in subSaharan Africa," Lancet Glob. Heal., 2017

4. Buckle GC, Walker CLF, Black RE, "Typhoid fever and paratyphoid fever: Systematic review to estimate global morbidity and mortality for 2010.," J. Glob. Health, 2012

5. Stanaway JD et al., "The global burden of typhoid and paratyphoid fevers: a systematic analysis for the Global Burden of Disease Study 2017," Lancet Infect. Dis., 2019

6. Mogasale $V$ et al., "Burden of typhoid fever in low-income and middle-income countries: $A$ systematic, literature-based update with risk-factor adjustment," Lancet Glob. Heal., 2014

7. Maurice J, "A first step in bringing typhoid fever out of the closet," Lancet, vol. 379, no. 9817, pp. 699-700, Feb. 2012

8. Blackstock SJ, Sheppeard VK, Paterson JM, Ralph AP (2013) Erratum to: Typhoid and paratyphoid fever in Western Sydney Local Health District, NSW, January? June 2011. N S W Public Health Bull 
24(1):2

9. H. S. et al., "Illness in children after international travel: Analysis from the geosentinel surveillance network," Pediatrics. 2010

10. Gibani MM, Britto C, Pollard AJ, "Typhoid and paratyphoid fever: a call to action," Curr. Opin. Infect. Dis., 2018

11. Crump JA, "Progress in Typhoid Fever Epidemiology," Clin. Infect. Dis., 2019

12. Institute of Brussels Statistics and Analysis ("More than one in three inhabitants of Brussels is not Belgian - Brussels Express," 2018) [Online]. Available: https://brussels-express.eu/one-threeinhabitants-brussels-not-belgian/. [Accessed: 08-Apr-2019]

13. Sánchez-Vargas FM, Abu-El-Haija MA, Gómez-Duarte OG (2011) Salmonella infections: An update on epidemiology, management, and prevention. Travel Med Infect Dis 9(6):263-277

14. Gibani MM, Britto C, Pollard AJ (2018) Typhoid and paratyphoid fever. Curr Opin Infect Dis 31(5):1

15. Pommelet $\mathrm{V}$ et al., "Enteric fever among children: 50 cases in a French tertiary care centre," J. Travel Med., 2018

16. Britto C, Pollard AJ, Voysey M, Blohmke CJ, "An appraisal of the clinical features of pediatric enteric fever: Systematic review and meta-analysis of the age-stratified disease occurrence," Clinical Infectious Diseases. 2017

17. Organization WH, "Typhoid vaccines: WHO position paper, March 2018 - Recommendations.," Vaccine. 2018

18. Sinha A et al., "Typhoid fever in children aged less than 5 years," Lancet, 1999

19. Gibani MM, Britto C, Pollard AJ, "Typhoid and paratyphoid fever: A call to action," Current Opinion in Infectious Diseases. 2018

20. Farmakiotis D et al., "Typhoid fever in an inner city hospital: A 5-year retrospective review," Journal of Travel Medicine. 2013

21. Zhou K, Sauve LJ, Richardson SE, Lee Ford-Jones E, Morris SK, "Enteric fever in a multicultural Canadian tertiary care pediatric setting: A 28-year review," J. Pediatric Infect. Dis. Soc., 2017

22. Mogasale V, Ramani E, Mogasale VV, Park JY (2016) What proportion of Salmonella Typhi cases are detected by blood culture? A systematic literature review. Ann Clin Microbiol Antimicrob 15(1):1-8

23. Wijedoru L, Mallett S, Parry CM, "Rapid diagnostic tests for typhoid and paratyphoid (enteric) fever," Cochrane Database of Systematic Reviews. 2017

24. Kuvandik C, Karaoglan I, Namiduru M, Baydar I, "Predictive value of clinical and laboratory findings in the diagnosis of the enteric fever," New Microbiol., 2009

25. Matono T et al., "Role of classic signs as diagnostic predictors for enteric fever among returned travellers: Relative bradycardia and eosinopenia," PLoS One, 2017

26. Wibrow BA, Ho KM, Flexman JP, Keil AD, Kohrs DL, "Eosinopenia as a diagnostic marker of bloodstream infection in hospitalised paediatric and adult patients: A case-control study," Anaesth. Intensive Care, 2011 
27. Abidi K et al., "Eosinopenia, an early marker of increased mortality in critically ill medical patients," Intensive Care Med., 2011

28. Gulati S, Maheshwari A, "Dengue fever-like illnesses: How different are they from each other?," Scandinavian Journal of Infectious Diseases. 2012

29. Choo KE, Davis TME, Henry RL, Chan LP, "Serum C-reactive protein concentrations in Malaysian children with enteric fever," J. Trop. Pediatr., 2001

30. Van Puyvelde S, Wong V, Jacobs J, Cuypers WL, Deborggraeve S, Klemm EJ, "Fluoroquinolone resistance in Salmonella: insights by whole-genome sequencing," Microb. Genomics, vol. 4, no. 7, 2018

31. Hasan Z et al (2018) Emergence of an Extensively Drug-Resistant Salmonella enterica Serovar Typhi Clone Harboring a Promiscuous Plasmid Encoding Resistance to Fluoroquinolones and ThirdGeneration Cephalosporins. MBio 9(1):1-10

32. Waddington CS et al (2017) Evaluation of the Clinical and Microbiological Response to Salmonella Paratyphi A Infection in the First Paratyphoid Human Challenge Model. Clin Infect Dis 64(8):10661073

33. Gibani MM, Britto C, Pollard AJ (2018) Typhoid and paratyphoid fever. Curr Opin Infect Dis 31(5):440-448

\section{Supplementary Files}

This is a list of supplementary files associated with this preprint. Click to download.

- Appendix1.docx

- Appendix2.docx

- Appendix3.docx

- Book2.xlsx 